\title{
A Novel TTC7A Deficiency Presenting With Combined Immunodeficiency and Chronic Gastrointestinal Problems
}

Sharafian $\mathrm{S}^{1}$, Alimadadi $\mathrm{H}^{2}$, Shahrooei $\mathrm{M}^{3,4}$, Gharagozlou $\mathrm{M}^{1}$, Aghamohammadi $\mathrm{A}^{1,5}$, Parvaneh $\mathrm{N}^{1,5}$

${ }^{1}$ Division of Allergy and Clinical Immunology, Department of Pediatrics, Tehran University of Medical Sciences, Tehran, Iran ${ }^{2}$ Division of Gastroenterology, Department of Pediatrics, Tehran University of Medical Sciences, Tehran, Iran

${ }^{3}$ Department of Microbiology and Immunology, Laboratory of Clinical Bacteriology and Mycology, KU Leuven, Leuven, Belgium ${ }^{4}$ Specialized Immunology Laboratory of Dr. Shahrooei, Ahvaz, Iran

${ }^{5}$ Research Center for Immunodeficiencies, Tehran University of Medical Sciences, Tehran, Iran

J Investig Allergol Clin Immunol 2018; Vol. 28(5): 358-360 doi: $10.18176 /$ jiaci.0290

Key words: Tetratricopeptide repeat domain 7A (TTC7A). Combined Immunodeficiency. Gastrointestinal.

Palabras clave: Dominio de Repeticiones de Tetratricopeptides 7A (TTC7A). Inmunodeficiencia combinada. Gastrointestinal.

Tetratricopeptide repeat domain 7A (TTC7A) deficiency is a very rare and severe hereditary disorder [1,2]. Only a few cases have been reported to date. TTC7A, which is expressed in various cells, plays an important role in cellular processes such as cell polarity, gut and lymphocyte homeostasis, and development of T and B lymphocytes [3]. Mutations in this gene usually result in gastrointestinal defects and immunodeficiency. Variations in clinical manifestations have been observed in patients with TTC7A mutations. The most widely reported are combined immunodeficiency (CID) and gastrointestinal defects such as multiple intestinal atresia (MIA), diarrhea, and extensive enteropathy [2]. We report the case of a patient with gastrointestinal problems and immunodeficiency syndrome.

A 4-year-old girl born to consanguineous Iranian parents presented with recurrent pneumonia and chronic diarrhea. Her birth weight was $3 \mathrm{~kg}$. She was the first of 2 children, her junior being a healthy 2.5 -year-old brother. During the second year of life, she was hospitalized several times owing to recurrent pneumonia. She also experienced nonbloody and nongreasy diarrhea, which gradually worsened. At 39 months of age, she was admitted to hospital with pneumonia and severe diarrhea (10-12 times a day) that had led to a 4-kg weight loss. She was discharged after a 2-week stay in hospital with a prescription for elemental formula, since the diarrhea had not completely ceased.

In February 2017, her eyes and skin turned yellow. Therefore, she was hospitalized in the gastrointestinal department because of liver and gallbladder involvement, as well as persistent diarrhea. Liver biopsy showed cholestatic 
liver disease with significantly reduced bile ducts in portal areas. Consequently, she was diagnosed with vanishing bile duct syndrome and became a candidate for liver transplantation.

In July 2017, she was hospitalized because of lethargy and anorexia. Intravenous immunoglobulin (IVIG) was administered monthly because of hypogammaglobulinemia (IgG, $146 \mathrm{mg} / \mathrm{dL}$; IgM, $12 \mathrm{mg} / \mathrm{dL}$; IgA, $3 \mathrm{mg} / \mathrm{dL}$ ). She was hospitalized owing to diarrhea and anorexia once again in September 2017.

Endoscopy and biopsy of the duodenum and stomach revealed erythema in the distal third of the esophagus, a large bleeding ulcer in the cardia, erosive gastritis, and moderate chronic gastritis with focal activity. Pathological findings in the biopsy specimens from the duodenum and antrum suggested autoimmune enteropathy and chronic erosive gastritis, respectively. Histopathology also revealed Helicobacter pylori.

Furthermore, high-resolution computed tomography of the lung revealed hyperaeration, mosaic attenuation, diffuse bronchiectasis, and peribronchial thickening. Additionally, atelectasis and consolidation were observed in the right middle lobe segment. Pleural effusion was not detected. A chest CT scan indicated right parahilar and left upper lobe alveolar consolidation, along with bronchiectasis in the right middle lobe. The main pulmonary artery was dilated, and pulmonary hypertension was suspected.

Magnetic resonance imaging revealed normal findings for the liver, spleen, pancreas, gallbladder, and kidney, although fluid dilation caused by ileus was observed in the small bowel and colon.

The lymphocyte and immunoglobulin profiles (normal range adapted from reference [4]) were as follows: T-cell subsets, CD3, 1780 (1400-3700) cells/ $\mu \mathrm{L} ;$ CD4, 593 (7002200) cells/ $\mu \mathrm{L} ; \mathrm{CD} 8,333.7$ (490-1300) cells/ $\mu \mathrm{L} ; \mathrm{B}-\mathrm{cell}$ subsets, CD19, 185.5 (390-1400) cells/ $\mu \mathrm{L} ; \mathrm{IgM}, 84 \mathrm{mg} / \mathrm{dL}$; IgG, $933 \mathrm{mg} / \mathrm{dL} ; \mathrm{IgA}, 8 \mathrm{mg} / \mathrm{dL}$. These findings indicated T-cell lymphopenia (CD4 and CD8 deficiency) and B-cell lymphopenia. Despite the low levels of immunoglobulins, the evaluation of the antibody response to diphtheria and tetanus was normal. A delayed-type hypersensitivity test with tetanus toxoid and a PPD test were performed to evaluate the function of cell-mediated immunity. The results of these tests indicated dysfunction of cellular immunity.

Whole exome sequencing revealed a novel homozygous mutation in the TTC7A gene (NM 001288951: exon17: c.1955delA). This took the form of a frameshift deletion mutation (p.Q652fs) that is likely pathogenic based on most predictors. Sanger sequencing confirmed that the patient was homozygous and her parents were heterozygous for this mutation.

The clinical manifestations, laboratory findings, and genetic study revealed gastrointestinal defects and immunodeficiency syndrome. Unfortunately, at the age of 4 years, the patient died of hepatic encephalopathy when she was admitted for liver transplantation.

We report the case of a patient with a novel $T T C 7 \mathrm{~A}$ mutation. To the best of our knowledge, there have been no previous reports of a frameshift deletion mutation in exon 17. Lawless et al [1] reported mutations of exon 17 in 3 patients, 2 of whom had MIA-CID, while common variable immunodeficiency accompanying enteropathy was reported in the third. Therefore, a missense mutation was identified in 2 patients and skipping of exon 17 in the TTC7A gene in 1 patient [1].

Phenotypic variation of $T T C 7 A$ mutations complicates timely diagnosis, with the result that different therapeutic procedures are required [5]. As stated in the literature review, up to three-quarters of patients with TTC7A mutations developed a CID phenotype. This abnormality stems from thymus failure and, consequently, defective lymphocyte development [5]. Despite the CD4 and CD8 deficiency in the patient we report, the T-cell count $\left(\mathrm{CD}^{+}\right)$was normal. This discrepancy could be attributed to the presence of a doublenegative $\mathrm{T}$-cell population $\left(\mathrm{CD}^{+} \mathrm{CD}^{-} \mathrm{CD}^{-}\right)$. It has been suggested that these double-negative $\mathrm{T}$ cells are involved in regulation of T-cell activation [6].

Recurrent diarrhea and enteropathy were major gastrointestinal problems in the present case. However, unlike some patients with TTC7A deficiency, this case indicated no evidence of intestinal atresia. It has been suggested that the absence of TTC7A protein could augment Rho kinase activity, and thereby growth, cell polarity, differentiation of intestinal cells, and cell homeostasis [3].

Even though most patients with $T T C 7 A$ mutations die in the early months of their lives [7], the initial symptoms of the present patient manifested in the second year of life. As reported by Fernandez et al [7], in addition to gastrointestinal effects and immunodeficiency, patients with $T T C 7 A$ mutation could also show dysfunctions of other organs such as the thymus, liver, and lungs. We identified pulmonary and liver involvement in the case we report.

Syndromic diarrhea (tricho-hepato-enteric syndrome) is another autosomal recessive disease that shares clinical manifestations - diarrhea and immunodeficiency — with MIACID. This rare syndrome is the result of a defect in the TTC37 and SKIVL2 genes. Patients with the syndrome manifest other symptoms such as facial dysmorphism and hair, skin, and liver abnormalities [8].

In conclusion, patients with gastrointestinal manifestations and immunodeficiency should be evaluated for mutations in TTC7A, TTC37, and SKIVL2 [8,9]. The severity of this abnormality, its variable phenotypes, and overlapping syndromes require advanced diagnostic measures such as whole exome sequencing to differentiate between alternatives and make a timely diagnosis.

\section{Funding}

The authors declare that no funding was received for the present study.

\section{Conflicts of Interest}

The authors declare that they have no conflicts of interest.

\section{References}

1. Lawless D, Mistry A, Wood PM, Stahlschmidt J, Arumugakani G, Hull M, et al. Bialellic Mutations in Tetratricopeptide 
Repeat Domain 7A (TTC7A) Cause Common Variable Immunodeficiency-Like Phenotype with Enteropathy. J Clin Immunol. 2017;37(7):617-22.

2. Yang $W$, Lee $P P$, Thong MK, Ramanujam TM, Shanmugam $A$, Koh MT, et al. Compound heterozygous mutations in TTC7A cause familial multiple intestinal atresias and severe combined immunodeficiency. Clin Genet. 2015;88(6):542-9.

3. Bigorgne $A E$, Farin $H F$, Lemoine $R$, Mahlaoui $N$, Lambert $N$, Gil M, et al. TTC7A mutations disrupt intestinal epithelial apicobasal polarity. J Clin Invest. 2014;124(1):328-37.

4. Shearer WT, Rosenblatt HM, Gelman RS, Oyomopito R, Plaeger S, Stiehm ER, et al. Lymphocyte subsets in healthy children from birth through 18 years of age: the Pediatric AIDS Clinical Trials Group P1009 study. J Allergy Clin Immunol. 2003 Nov; 112(5):973-80.

5. Lien R, Lin YF, Lai MW, Weng HY, Wu RC, Jaing TH, et al. Novel Mutations of the Tetratricopeptide Repeat Domain 7A Gene and Phenotype/Genotype Comparison. Front Immunol. 2017;8:1066.

6. Bhatnagar N, Girard PM, Lopez-Gonzalez M, Didier C, Collias $\mathrm{L}$, Jung $\mathrm{C}$, Bollens $\mathrm{D}$, et al. Potential Role of $\mathrm{V} \delta 2+\gamma \delta \mathrm{T}$ Cells in Regulation of Immune Activation in Primary HIV Infection. Front Immunol. 2017;8:1189.

7. Fernandez I, Patey N, Marchand V, Birlea M, Maranda B, Haddad $E$, et al. Multiple intestinal atresia with combined immune deficiency related to TTC7A defect is a multiorgan pathology: study of a French-Canadian-based cohort. Medicine (Baltimore). 2014;93(29):e327.

8. Neves JF, Afonso I, Borrego L, Martins C, Cordeiro Al, Neves $C$, et al. Missense mutation of TTC7A mimicking trichohepato-enteric (SD/THE) syndrome in a patient with very-early onset inflammatory bowel disease. Eur J Med Genet. 2018 Apr;61(4):185-8.

9. Guana R, Garofano S, Teruzzi E, Vinardi S, Carbonaro G, Cerrina $A$, et al. The complex surgical management of the first case of severe combined immunodeficiency and multiple intestinal atresias surviving after the fourth year of life. Pediatr Gastroenterol Hepatol Nutr. 2014;17(4):257-62.

I Manuscript received April 28, 2018; accepted for publication June 28, 2018.

Nima Parvaneh

Division of Allergy and Clinical Immunology, Department of Pediatrics Children's Medical Centre, No 62 Gharib St 14194 Tehran, Iran. E-mail: nparvaneh@tums.ac.ir 